https://doi.org/10.22319/rmcp.v11i2.5173

Nota de investigación

\title{
Factores asociados al decomiso de hígados positivos a Fasciola sp en una zona
} endémica del sureste de México

Nadia Florencia Ojeda-Robertos ${ }^{\text {a }}$

Roberto González-Garduño ${ }^{\text {b }}$

Santiago Cornelio-Cruz ${ }^{\text {a }}$

Jorge Alonso Peralta-Torres ${ }^{\text {a }}$

Carlos Luna-Palomera ${ }^{\mathrm{a}}$

Carlos Machain-Williams ${ }^{\mathrm{c}}$

Heliot Zarza ${ }^{\mathrm{d}}$

Oswaldo Margarito Torres-Chablé ${ }^{\mathrm{a}}$

Enrique Reyes-Novelo ${ }^{c}$

Carlos Baak-Baak ${ }^{\mathrm{c}}$

Alfonso Chay-Canul ${ }^{\text {** }}$

${ }^{a}$ Universidad Juárez Autónoma de Tabasco. División Académica de Ciencias Agropecuarias. Villahermosa, Tabasco, México.

b Universidad Autónoma Chapingo, URUSSE. Teapa, Tabasco, México.

c Universidad Autónoma de Yucatán. Centro Regional de Investigaciones "Dr Hideyo Noguchi” Mérida, Yucatán.

${ }^{\mathrm{d}}$ Universidad Autónoma Metropolitana. Departamento de Ciencias Ambientales, CBS, Unidad Lerma, Estado de México, México. 
Autor de correspondencia: aljuch@hotmail.com

\section{Resumen:}

El objetivo fue determinar la frecuencia por decomiso de hígados con daños atribuidos a la presencia de Fasciola sp y los factores de riesgo asociados. Se realizó un estudio prospectivo observacional en el que se efectuaron visitas diarias durante un periodo de 12 meses, a un rastro municipal en la zona Sierra del estado de Tabasco. El $25.8 \%$ de los hígados fueron positivos a la presencia del parásito, siendo el decomiso por sexo igual para machos y hembras $\left(\mathrm{X}^{2}=0.011, \mathrm{gl}=\right.$ 1, $P<0.05)$. La mayor proporción de decomiso, se concentró en la época de lluvias (9.36 \%). Se concluye que la prevalencia de la fasciolosis en la zona de Jalapa no ha disminuido en los últimos años y está relacionada con el origen del animal. La fasciolosis es una enfermedad que debe ser monitoreada para detectar los factores que permiten su permanencia en una región geográfica, con el fin de poder establecer y plantear medidas estratégicas de control y de prevención adaptadas a las condiciones particulares de las zonas endémicas.

Palabras clave: Decomiso, Fasciola sp., Hígado, Tabasco.

Recibido: 03/12/2018

Aceptado: 02/04/2019

La fasciolosis es una enfermedad parasitaria zoonótica, causada por la presencia de trematodos del género Fasciola sp en los conductos hepáticos de rumiantes ${ }^{(1,2)}$. Es una enfermedad de importancia económica, ya que afecta especies productivas como el ganado bovino, ovino, equino y porcino además de animales silvestres ${ }^{(3)}$. Se estima que los costos económicos asociados a su presencia ascienden a los 3 millones de dólares en todo el mundo ${ }^{(4)}$. En México, las pérdidas ascienden a 130 mil dólares ${ }^{(5)}$, además de que es una enfermedad parasitaria, que está incluida en la lista de investigación prioritaria de las enfermedades tropicales negadas ${ }^{(1)}$. En los últimos años, la prevalencia de la fasciolosis en animales domésticos y en seres humanos, se ha incrementado, debido al cambio climático, al movimiento de animales de unas áreas a otras, así como al tráfico de viajeros y de migrantes ${ }^{(6,7)}$.

En Tabasco, una de las principales actividades agropecuarias es la ganadería bovina, basado en sistemas de pastoreo intensivo o extensivo, condición que favorece la presencia y transmisión de la enfermedad en los rumiantes de la región. La enfermedad se produce cuando los animales, 
ingieren los estadios infectantes (metacercarias), que se localizan enquistadas en los pastos, por lo que de ahí la importancia de esta enfermedad en el sistema de producción.

La fasciolosis ha sido reportada como un parasitismo común en la población animal con una prevalencia estatal del $19.7 \%{ }^{(8)}$, encontrándose variación dependiendo de la zona y de las características de cada región. Rangel y Martínez ${ }^{(8)}$, identificaron zonas de alta, mediana y baja prevalencia, lo cual clasifica al estado en una zona endémica de la enfermedad junto con Chiapas y Veracruz.

Ante la falta de una técnica diagnóstica sensible y específica para la detección de animales positivos, una forma de determinar la prevalencia verdadera, es mediante el monitoreo en rastros, ya que puede proporcionar información epidemiológica relevante a relativamente bajo $\operatorname{costo}^{(9)}$, por lo que, el análisis de esta información puede ser utilizada para determinar el comportamiento y significancia de la enfermedad en una región ${ }^{(9)}$. La inspección sanitaria es un procedimiento que se realiza rutinariamente en el proceso de sacrificio de bovinos que son destinados para carne de abasto para consumo humano y es una pieza clave para realizar estudios epidemiológicos que permitan vislumbrar el escenario de la enfermedad. La presencia de hígados positivos a Fasciola es causa de decomiso inmediato, sin embargo, aún falta por conocer más acerca de los factores que están relacionados con la presencia de la enfermedad. El objetivo del presente trabajo fue determinar la prevalencia en rastro, así como los factores asociados al decomiso de hígados en una zona endémica del sur de México.

El trabajo se realizó en el Estado de Tabasco, en el rastro municipal de Jalapa, el cual está ubicado en la región del Rio Grijalva, subregión Sierra del estado. La zona se caracteriza por tener un clima cálido húmedo con lluvia todo el año (Af), según la clasificación climática de Koppen modificada por García ${ }^{(10)}$. Se registra una temperatura media de $24.9{ }^{\circ} \mathrm{C}$ y precipitación pluvial anual de $3,711 \mathrm{~mm}^{(11)}$. El municipio de Jalapa cuenta con cuerpos de agua que están conformados por dos grandes ríos, el Rio de la Sierra y Puente Grande, así como con arroyos y lagunas, este complejo sistema orográfico durante las épocas de lluvias se desborda causando inundación en la zona.

Se realizó un estudio prospectivo observacional durante un periodo de 12 meses (enero a diciembre 2014) en el que se realizaron visitas diarias al rastro municipal. Las inspecciones se realizaron siguiendo los procedimientos de sacrificio del rastro, los cuales se apegan a las normas de sacrificio NOM 033-ZOO-1995. Después del sacrifico y una vez que el paquete visceral fue separado de la canal, se procedió a la inspección de los órganos, la cual se realizó según la norma zoosanitaria NOM-194-SSA-2004, que especifica que, la revisión de las vísceras se realiza en búsqueda de presencia de parásitos y estos deben estar ausentes. 
Cuando se detectó la presencia de un hígado con lesiones sugerentes a la presencia del parásito, se separó para ser inspeccionado minuciosamente. Los hígados decomisados se inspeccionaron en búsqueda de lesiones y de parásitos adultos o de formas migratorias, para lo cual se realizaron incisiones en el tejido parenquimatoso y se clasificaron en positivos y negativos a la presencia de Fasciola. Los positivos fueron los que cumplieron cualquiera de las condiciones: la presencia de lesiones macroscópicas como abscesos y engrosamiento de las paredes de los conductos biliares, la presencia de los parásitos adultos o en desarrollo vivos o muertos en los conductos hepáticos o en el parénquima. Un hígado negativo fue aquel en que no se encontraron lesiones, ni parásitos.

De cada animal, independientemente si fueron positivos o no, se colectaron los datos que incluyeron fecha de sacrificio (día y mes), sexo (macho o hembra), localidad de procedencia del introductor (localidad) y decomiso del hígado (positivo o negativo). Se calculó la cantidad y frecuencia de hígados decomisados en el periodo de estudio, por mes y por época del año. La frecuencia de hígados decomisados se calculó usando la fórmula para determinar prevalencia descrita por Thrusfield ${ }^{(12)}$ y fue expresado como el porcentaje del número total de bovinos sacrificados respecto del número de animales que ingresaron al rastro para sacrificio. Los datos se agruparon por sexo, mes de decomiso, época del año (lluvia, seca y norte) y por población de procedencia para determinar su asociación con el decomiso de hígados positivos a Fasciola sp. En el caso de los datos de los meses, estos se agruparon en tres epocas, en secas (marzo-junio), lluvias (julio-octubre) y nortes (noviembre-febrero).

Los datos agrupados por sexo se analizaron por medio de Ji cuadrada en una tabla de contigencia 2 x 2. Para detectar diferencias por mes, los datos se analizaron por medio de Kruskal-Wallis. Se realizaron análisis de asociación, por medio de una prueba de correspondencia, para la época del año y localidad. Se utilizaron tablas de contingencia 3 × 2 y 27 x 2 respectivamente. Los análisis se realizaron usando el programa estadístico SPSS versión 8.

La cantidad de animales que ingresaron al rastro municipal durante el año varió mensualmente, entre 42 a 108 para el mes enero y mayo respectivamente. Aun así y dentro de este contexto, el número de hígados positivos que fueron detectados fue de 278 , lo que representó el $25.8 \%$ del total de decomisados (Cuadro 1), lo cual se encuentra dentro del rango de prevalencia reportada para el Estado de Tabasco ${ }^{(8,13,14)}$ porcentaje que sigue sin cambio aparente desde los primeros estudios realizados en rastros tabasqueños ${ }^{(8)}$. 
Cuadro 1: Número de bovinos sacrificados e hígados decomisados durante un año en un rastro municipal de la zona sureste, Jalapa, Estado de Tabasco, México

\begin{tabular}{lccccc}
\hline \multicolumn{5}{c}{ Hígados decomisados } \\
Bovinos & n & Positivos & Negativos & \% de Positivos & Valor de $\boldsymbol{P}$ \\
\hline Hembras & 1025 & 264 & 761 & 25.7 & \\
Machos & 53 & 14 & 39 & 26.4 & 0.005 \\
Total & 1078 & 278 & 800 & 25.8 & \\
\hline
\end{tabular}

Los factores de riesgo, que han sido ligados a la prevalencia de la enfermedad en los bovinos son los relacionados con el medio ambiente, como la temperatura y humedad, medios hídricos como los ríos; la presencia del hospedero intermediario y los factores ligados al hospedero definitivo como son la edad, raza, especie animal y el sistema de explotación y manejo de la alimentación. Así mismo, la ubicación del rancho y las condiciones microclimáticas predominantes en el área de pastoreo de los animales son de gran importancia en la epidemiología de la enfermedad ${ }^{(15)}$.

En este estudio, independientemente de la cantidad de animales que llegaron al rastro, la probabilidad de que los machos fueran positivos fue la misma que para las hembras, ya que no existió relación entre el decomiso de hígados positivos a la presencia del parasito y el sexo $\left(\mathrm{X}^{2=} 0.011, \mathrm{gl}=1, P<0.05\right)$, lo cual coincide con lo reportado por Ticona ${ }^{(16)}$ quien menciona que el sexo no fue un factor de riesgo para la presencia de la enfermedad en Perú, siendo la edad un factor de riesgo más asociado a la presencia de la enfermedad ${ }^{(17,18)}$.

Un factor que puede ser confundido con el sexo y la edad, es el sistema de producción en el que son criados los animales, ya que, por lo general, son las hembras las que son criadas fundamentalmente bajo condiciones de pastoreo ${ }^{(19)}$, además de que permanecen por un periodo mayor de tiempo en los ranchos, situación que difiere de los machos que generalmente son vendidos para ser engordados bajo sistemas intensivos; sin embargo, los machos también son susceptibles de parasitarse cuando son manejados bajo sistema de pastoreo.

En el presente trabajo, la variación mensual en el decomiso de positivos varió de 3.6 a $12.3 \%$, y se encontró que en todos los meses se detectó la presencia de Fasciola sp. encontrándose diferencias entre meses $\left(X^{2}=51.918, \mathrm{gl}=11, P=0.000\right)$. Sin embargo, el mes con mayor porcentaje de higados positivos fue febrero con $17.3 \%$ y el menor fue noviembre con $3.6 \%$ (Figura 1). La época con mayor porcentaje de hígados positivos fue la de lluvias con un $9.36 \%$ (junio a octubre), seguida por la seca con $8.34 \%$ (marzo-mayo) y la de norte con un $8.07 \%$ (noviembre a febrero). Se determinó que existió relación entre la época de muestreo y el decomiso $\left(X^{2}=6.511\right.$, gl 2, $P=0.039)$. Lo anterior, coincide con lo señalado por Feunmayor y Ojeda-Robertos ${ }^{(14,19)}$ quienes encontraron relación entre la época del año y la presencia de animales postivos a 
Fasciola, ambos autores utilizaron la técnica de sedimentación para determinar la prevalencia en animales bajo pastoreo.

Figura 1: Porcentaje de hígados positivos a Fasciola sp por mes y por época del año

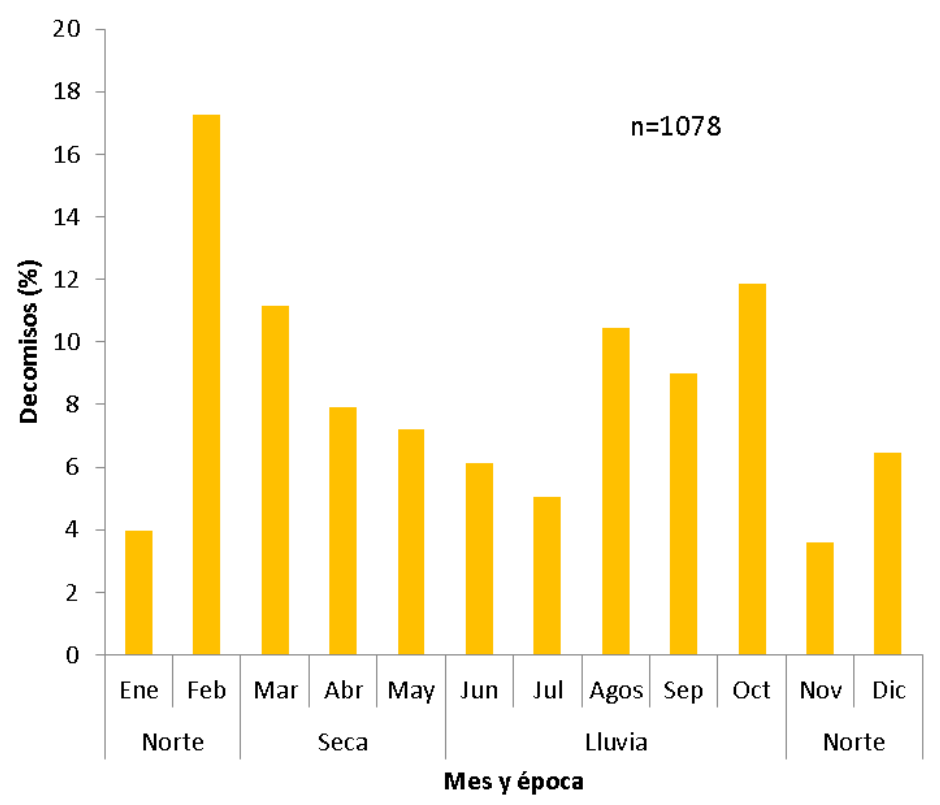

En este estudio, la detección de los hígados positivos y con daños hepáticos posmortem, es la evidencia de que los animales en algún momento de su vida estuvieron en contacto con el estadio infectante de la Fasciola sp. y que adquirieron la enfermedad cuando las condiciones medioambientales externas fueron propicias para el desarrollo de estas fases exógenas en los ranchos donde se criaron. Lo cual, se confirma al encontrar los parásitos con diferentes estadíos de desarrollo en los conductos hepáticos; sin embargo, no se realizó un conteo de los estadios de desarrollo parasitario encontrados en los hígados positivos.

La influencia de la época es determinante para el mantenimiento de las fases exógenas del trematodo, ya que se relaciona directamente con el desarrollo y sobrevivencia del hospedero intermediario y las fases infectantes del parásito ${ }^{(20) .}$ La Fasciola hepatica posee un ciclo complejo que incluye la presencia de dos hospederos obligatorios, el definitivo (rumiantes y humano) y el intermediario (moluscos del género Fossaria y Pseudosuccinea de la familia Lymnaeidae).

En ambos hospederos, la eliminación de los estadios parasitarios permiten que se cumpla con el ciclo del vida del parásito, ya que los huevos, se eliminan con las heces del hospedero definitivo al medio ambiente, mientras que las cercarias son expulsadas por el caracol, las cuales se enquistan sobre la vegetación acuática o lado de los cuerpos de agua o el pasto, transformándose en metacercarias (fase infectante) $)^{(21,22)}$. 
La metacercaria, al ser ingerida junto con los pastos o agua contaminada, por el hospedador definitivo, se desarrolla hasta el estadio juvenil, la cual tiene la capacidad de migrar atravezando la pared intestinal, alojándose posteriormente en la cavidad abdominal, peritoneo, cápsula de Glisson, y el parénquima hepático; luego se establece en los conductos biliares, alcanza su etapa adulta y la madurez sexual para dar inicio a la ovoposición de huevos fértiles, los cuales son eliminados junto con las heces al medio externo ${ }^{(15)}$.

Al examen postmortem, se detecta el hígado con hipertrofia, hemorrágico, con diferentes grados de fibrosis, calcificación e hiperplasia de los conductos biliares, así como la presencia de las formas parasitarias en el tejido hepático, lo cual es un motivo de decomiso independientemente del grado de afectación del hígado ${ }^{(23)}$.

En un estudio realizado en el noreste de Nigeria en el continente Africano, se determinó el grado de daño de hígados decomisados por la presencia de Fasciola y obtuvieron que la mayor frecuencia fue de hígados con daños severos, $(55.3 \%)$ seguido por daños moderados, esos daños incluyeron desde fibrosis hasta daño total del hígado y atrofia ${ }^{(24)}$. En este trabajo se realizó la descripción del grado del daño de los hígados positivos, lo cual pudiera ser un tema a investigar para futuros estudios.

En el presente trabajo, la época del año en la que se detectó mayor cantidad de hígados decomisados se relacionó posiblemente con la mayor cantidad de animales que provinieron de una localidad donde las prevalencias y las probabilidades de enviar a un animal positivo al rastro son más altas, debido a que las condiciones del lugar favorecen la presencia de los estadios infectantes dadas las condiciones propias del lugar (suelos inundables, cercanía con el río y mantos de agua).

La procedencia del total de decomisos $(\mathrm{n}=1,078)$, independientemente de la causa, se distribuyeron en 27 poblados pertenecientes al municipio de Jalapa. Del total de localidades registradas, siete contribuyeron con más de la mitad del total de positivos $(89.2 \%, 248 / 278)$, diez localidades aportaron el $10.8 \%$ restante $(30 / 278)$ y en las diez restantes no se detectaton decomisos. El aporte de las siete localidades varió de 16 a 45 hígados positivos, en las segundas de 1 a 7 hígados positivos y en las 10 restantes cero. De cada poblado, la cantidad de animales que se recepcionaron para sacrificio fue variable y dependió de las necesidades de los productores para enviar animales a sacrificio.

La prevalencia ajustada a la población fue del $16.19 \%$ (Huapacal) para la más alta y de $5.40 \%$ para la más baja (Figura 2); se determinó que existió relación entre las localidades de procedencia y la presencia de hígados positivos $\left(X^{2}=59.621\right.$, gl $\left.26 P=0.000\right)$. El origen de los animales es un factor importante que está relacionado con el decomiso, ya que las condiciones regionales 
ambientales de la localidad, así como el manejo en el sistema producción, se reflejan en el número de animales positivos, y es muy problable que los animales de estas regiones hayan tenido un mayor contacto con los estadios infectantes del parásito por las condiciones particulares de la región de crianza ${ }^{(24) .}$

Figura 2: Frecuencia de decomiso de hígados positivos por población en el rastro municipal de Jalapa, Tabasco, México

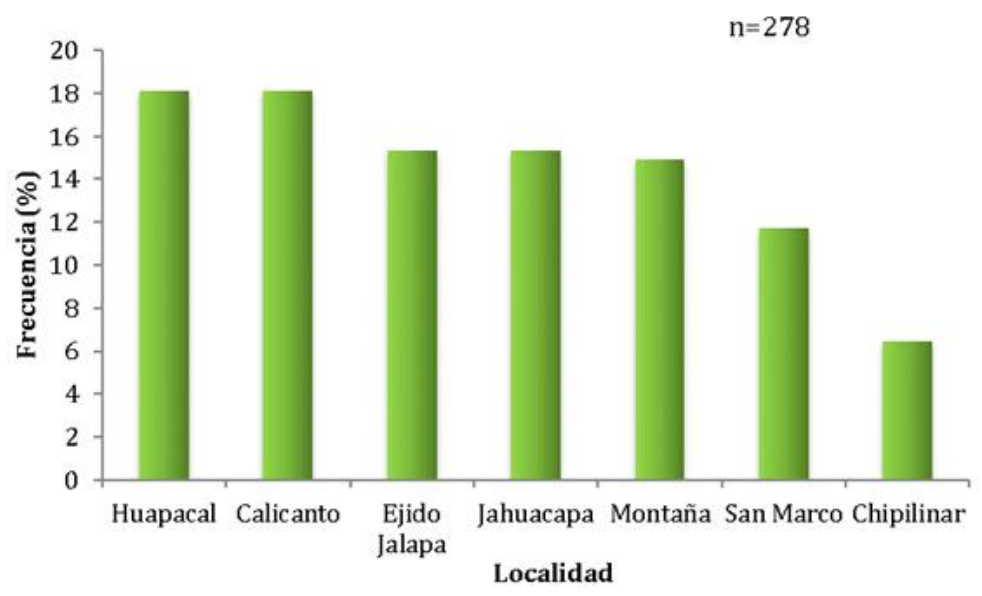

Se concluye que la prevalencia de la fasciolosis en la zona de Jalapa, Tabasco, no ha disminuido en los últimos años, a pesar de las medidas de control tradicionales, entre las que se encuentran el uso de fasciolicidas para el control de los estadios inmaduros y adultos, así como la restricción para pastorear zonas cercanas a los ríos, para evitar la ingestión de las metacercarias. La prevalencia de la enfermedad está relacionada con la localización u origen del animal, aunque existen otros factores que influencian la presencia de la enfermedad que deben ser estudiados, entre los que se encuentran, el tipo y sistema de producción pecuaria, el manejo de los productos fasciolicidas y las condiciones microclimáticas de cada lugar donde se crían a los animales.

La fasciolosis como enfermedad que afecta animales y humanos debería ser monitoreada para aumentar el conocimiento de su epidemiología para proponer medidas de control y prevención dependiendo de las condiciones microclimáticas. Ante los cambios climáticos, se recomienda un monitoreo estatal y nacional que pudiera aportar información para entender mejor la ecología de la enfermedad y la presentación de la Fasciolosis en animales rumiantes.

\section{Agradecimientos}

Al fondo PFI 2013-UJAT por el financiamiento del proyecto clave UJAT-2013-IA-10. En memoria del Pasante de MVZ Santiago Cornelio Cruz, quien fue pieza clave y determinante en la 
gran labor de la colecta diaria de los datos en el rastro. Al MVZ Juan Felipe Jiménez por su gran apoyo, disposición y paciencia para ayudarnos en el rastro. Al Laboratorio de Parasitología Animal del Centro de Investigación Ciencias Agropecuarias-UJAT por las facilidades otorgadas durante las inspecciones de los hígados.

\section{Literatura citada:}

1. World Health Organization. Research priorities for zoonosis and marginalized infections. Technical Reports Series 2013.

2. Arafa WM, Hassan AI, Snousi SAM, El-Dakhly, Holman PJ, Craig TM, Aboelhadid SM. Fasciola hepatica infections in cattle and the freshwater snail Galba truncatula from Dakhla Oasis, Egypt. J Helminthol 2017; doi: https://doi.org/10.1017/S0022149X17000086..

3. Pérez Creo A, Béjar JP, Díaz CM, López A, Prieto M, Viña V, Martinez, Diez-Baños P, Ubeira FM, Morrondo P. Fasciola hepatica in sheep from north-western Spain. Risk factor analysis using a capture ELISA (MM3 SERO). Small Ruminant Res 2016;45:103-106.

4. Food and Agricultural Organization of the United Nations. Diseases of domestic animals caused by liverflukes: epidemiology, diagnosis and control of Fasciola, Paramphistome, Dicroceoelium, Eurytrema and Schistosome infections of ruminants in developing countries. Rome: FAO. 1994.

5. Rodríguez-Vivas RI, Grisi L, Pérez de León AA, Silva Villela H, Torres-Acosta JFJ, Fragoso Sánchez $\mathrm{H}$, et al. Evaluación del impacto económico potencial de los parásitos del ganado bovino en México. Rev Mex Cienc Pecu 2017;8:61-76.

6. Mas-Coma S, Esteban JG, Bargues MD. Epidemiology of human fascioliasis: a review and proposed new classification. Bulletin WHO 1999;77:340-346.

7. Ashrafi K, Bargues MD, O`Nell S, Mas-Coma S. Fascioliasis: A worldwide parasitic disease of importance in travel medicine. Travel Med Infect Dis 2014;12:636-649.

8.-Rangel Ruiz LJ, Martínez DE. Pérdidas económicas por decomiso de hígados y distribución geográfica de Fasciolosis bovina en el Estado de Tabasco. Vet Méx 1994;25:327-331.

9. Roberts JA. Suhardono S. Approaches to the control of fasciolosis in ruminants. International J Parasitol 1996;26:971-981. 
10. García E. Modificaciones al sistema de clasificación climática de Koppen. 5ta ed. Instituto Nacional de Geografía. UNAM. 2004.

11. De Dios OO. El medio ambiente tropical. En: Ecofisiología de los bovinos en sistemas de producción del trópico húmedo. Colección José N. Rovirosa. Edit. Universidad Juárez Autónoma de Tabasco, México. 2001.

12. Thrusfield M. Epidemiología veterinaria. México: Acribia Ed. Zaragoza. 1990.

13. Rangel-Ruiz LJ, Izquierdo MR, Nogeira BG. Bovine fasciolosis in Tabasco, Mexico. Vet Parasitol 1999;81:119-127.

14. Ojeda-Robertos NF, Medina-Reynes U, Garduza-Arias G, Rangel-Ruiz LJ. Dinámica de excreción de huevos de Fasciola hepática y Paramphistomum spp en ganado bovino de Tabasco. Ecosist Recur Agropec 2014;1:73-80.

15. Acha P, Szyres B. Zoonosis y enfermedades transmisibles comunes al hombre y a los animales. 3a ed. Washington: OPS.; 2003.

16. Ticona S, Daniel, Chávez V, Amanda Chavera C, Alfonso Casas V, Gina Li E. Prevalencia de Fasciola hepática en bovinos y ovinos de Vilcashuamán, Ayacucho. Rev Invest Vet Perú 2010;168-174.

17. Recalde-Reyes DP, Padilla Sanabria L, Giraldo Giraldo MI, Toro Segovia LJ, González MM, Castaño OJC. Prevalencia de Fasciola hepática en humanos y bovinos en el departamento del Quindío-Colombia 2012-2013. Infectio 2014;18:153-157.

18. Sandoval SE, Medina R, Alfonso PS. Prevalencia de la distomatosis hepática en 4 unidades Agroecológicas del Bajo Tocuyo, Estado Falcón. Vet Trop 1989;14:43-51.

19. Fuenmayor A, Simoes D, González R, Chirinos A. La Distomatosis hepática y su asociación con los factores de riesgo en los Municipios Mara y Páez del estado Zulia, Venezuela. Rev Cient FCV-LUZ, 2000;103:183-190.

20. López-Villacís IC, Artieda-Rojas JR, Mera-Andrade RI, Muñoz-Espinoza MS, Rivera-Guerra VE, Cuadrado-Guevara AC, et al. Fasciola hepática: aspectos relevantes en la salud animal. J Selva Andina Animal Sci 2017;4(2):137-146. 
21. Rojo-Vázquez FA, Ferre-Pérez I. Parasitosis hepáticas: Fasciolosis. En: Parasitología Veterinaria. Cordero del Campillo M, Rojo VFA editores. Madrid, España: Mc Graw Hill. Interamericana 1999:260-272.

22. Carrada BT, Escamilla JR. Fasciolosis: revisión clínico-epidemiológica actualizada. Rev Mex Patol Clin 2005;52(2):83-96.

23. Mwabonimana MF, Kassuku AA, Ngowi HA, Mellau SB, Nonga HE, Karimuribo ED. Prevalence and economic significance of bovine fasciolosis in slaughtered cattle at Arusha abattoir, Tanzania. Tanzania Vet J 2009;26(2):68-74.

24. Karshima NS, Bata SL. Bobbo AA. Prevalence, risk factors and losses associated with Fasciolosis in slaughtered cattle in Bauchi, North-Eastern, Nigeria. Alexandria J Vet Sci 2016; 50:87-93. 